\title{
Statistics-based procedure for defect sizing and experimental evaluation of convection phenomena by using Video Pulsed Thermography
}

\author{
by F. Cernuschi ${ }^{1}$, N. Ludwig ${ }^{2}$ and P. Teruzzi ${ }^{2}$ \\ ${ }^{1}$ ENEL Research, Via Rubattino, 5420134 Milano Italy, E-mail Cernuschi@cram.enel.it \\ ${ }^{2}$ Università degli Studi di Milano, Istituto di Fisica Generale Applicata, Via Celoria, 1620133 Milano \\ Italy; E-mail: nicola.ludwig@mi.infn.it.
}

\begin{abstract}
In the frame of defect sizing procedures using pulse thermography, the time evolution of the Full Width Half Maximum (FWHM) of the temperature difference between a defect region and a sound one was studied both theoretically and experimentally in the simple case of a circular sub-surface defect. A mismatch between experimental results and theoretical simulations has been found. Possible explanations of this disagreement have been analysed. Moreover an extension of a sizing procedure (applied to circular defects) to irregular shaped defects is presented.
\end{abstract}

\section{Introduction}

Nowadays the main topic of research, as far as pulse thermography is concerned, is strongly related to the development of suitable inversion algorithms allowing defect sizing. Good reviews of theoretical models and sizing algorithms are reported in [1-2].

In some cases, the FWHM of the temperature difference profile (after the heating pulse) between defect region and the sound one in the simple case of circular sub-surface defects is indicated to be a good measure of the defect diameter [3,4].

This work mainly deals with the study of both the theoretical and the experimental time dependence of FWHM. This dependence has been analysed theoretically by studying both analytical and numerical 2D models that describe the time evolution of the temperature distribution of the sample surface when a circular shaped defect is present beneath the surface. Analogously FWHM as a function of time has been experimentally investigated in different conditions. Finally, a sizing procedure for irregularly-shaped defects is proposed.

\section{Sizing procedures}

\subsection{Remarks}

For flat bottom holes, a good measure of the defect diameter in single frequency $\omega$ thermal waves models and experiments, is given by the FWHM of the spatial profile of the thermal contrast (i.e. the temperature difference between defect region and the sound one) $[3,4]$. On the contrary, for a circular crack (in literature is reported only the case of a $10 \mathrm{~mm}$ diameter crack) it resulted that the defect's FWHM is of $0.54 \mu$ (where $\mu=\sqrt{2 \alpha / \omega}$ is the thermal diffusion length and $\alpha$ is the thermal diffusivity) is smaller than its true defect diameter [3]. This can be justified considering that a crack with finite dimensions allows the heat to propagate also in the material under it.

A Dirac pulse can be represented as a superposition of all frequencies. In order to extend the defect sizing algorithm onto pulsed thermography, it is necessary to pass from the frequency to time domain. Practically this means to substitute the thermal diffusion length $\mu$ with the effective one defined as [3]: 


$$
\mu_{e f f}=2 \sqrt{\alpha t}
$$

The simple equation connecting, the measured FWHM at different times with the true diameter $D_{\text {eff }}$ in transient thermography experiments is [3]:

$$
F W H M(t)=D_{\text {true }}-0.54 \mu_{\text {eff }}
$$

Eq. (2) is a straight line as a function of the square root of the time. Thus, for correct defect sizing it should be sufficient to consider the value obtained by extrapolating to $t=0$ the eq.(2) obtained by fitting the experimental data. Obviously, following this way of thinking, the FWHM of the thermal contrast due to a flat bottom hole should be time-independent.

On the other hand, a specific study of the theoretical time evolution of FWHM as foreseen by some different $2 \mathrm{D}$ models describing the thermal contrast (due to circular defects) on the sample surface during a pulse thermography experiment has been performed [5]. In particular, the FWHM has been first evaluated as a function of time $t$ in the case of a flat bottom hole for the models proposed by Almond et al. [3, 6], and by Crowther et al. [7]. FWHM has been computed solving numerically the following analytical integral equations both for single and multiple reverberations (represented by the sum index $m$ ) [5-7]:

$$
\begin{gathered}
C(x, t)=\frac{2 I}{\pi \kappa} \int_{0}^{a} d r \int_{0}^{\pi} d \theta \sum_{m=0}^{+\infty} \frac{\exp \left(-((2 m+1) l+R)^{2} / 4 \alpha t\right.}{2 \sqrt{\pi \alpha t^{3}}} \cdot \frac{l+R}{R} \cdot r \\
C(x, l) \cong-\frac{1}{2 \pi \varepsilon \sqrt{\pi}} \frac{\partial}{\partial z} \int_{-\infty}^{+\infty} d x^{\prime} \int_{-\infty}^{+\infty} d y^{\prime} \times\left.\sum_{m=-\infty}^{+\infty} \frac{\exp \left(-\left[\sqrt{\left(x-x^{\prime}\right)^{2}+\left(y-y^{\prime}\right)^{2}+(2 m+1)^{2} l^{2}}+z\right]^{2} / 4 \alpha t\right)}{\sqrt{\left(x-x^{\prime}\right)^{2}+\left(y-y^{\prime}\right)^{2}+(2 m+1)^{2} l^{2}}}\right|_{==l} \times f\left(x^{\prime}, y^{\prime}\right)
\end{gathered}
$$

where $\alpha=\frac{\kappa}{\rho C}, \varepsilon=\sqrt{\kappa \rho C}$ ( $\kappa$ thermal conductivity, $\rho$ density and $C$ specific heat) and $/$ are the thermal diffusivity, the thermal effusivity and the energy density of the heating pulse, respectively. Where $l, a$, are depth and radius of the defect and $R=\left(x^{2}+l^{2}+r^{2}-2 r x \cos \theta\right)^{1 / 2}$. $(x, y, z)$ and $\left(x^{\prime}, y^{\prime}, z^{\prime}\right)$ refer to cartesian co-ordinates systems centered on the defect centre and lying on the sample surface and on the defect plane respectively. The defect shape function in this case is:

$$
f\left(x^{\prime}, y^{\prime}\right)=\left\{\begin{array}{l}
1 \text { if } x^{\prime 2}+y^{\prime 2} \leq a^{2} \\
0 \text { if } x^{\prime 2}+y^{\prime 2}>a^{2}
\end{array}\right.
$$

In figure 1, the curves of the FWHM are shown as a function of square root of time evaluated using eqs. (3) and (4). In can be noted that in all cases the functions fitting these computed data appear to be parabola -like curves. On the contrary it would be expected to obtain FWHM versus square root of time data that was represented with a straight line parallel to the abscissa axis (remembering that the defect is a flat bottom hole). Experimental results, carried out on a set of ferritic steel samples containing flat bottom holes with two different diameters $(7 \mathrm{~mm}, 10 \mathrm{~mm})$ and at two depths $(0.5 \mathrm{~mm}$ and $1 \mathrm{~mm})$, have given the evidence (see Fig. 2) in all 3 cases of a linear trend of the FWHM in respect to the square root of time. In order to understand the effective influence on the FWHM trend of the material under the defect, a $10 \mathrm{~mm}$ hole at $1 \mathrm{~mm}$ depth was partially bunged using a cylinder of the same material as the sample. In this way the air gap thickness under the defect was reduced from infinity to some tenth of millimetres; moreover a nearly perfect thermal contact between walls of the defect and of the bung was assured. After this defect modification, the trend of FWHM versus. square root of time shows a slope higher than the previous case (see Fig. 2). This seems to be qualitatively in good agreement with eq. (2). It should be also noted that the 
FWHM extrapolation at time $t=0$ give, for all the defects a good estimation of their diameters. Thus the proposed method appears to be suitable for the correct sizing of circular defects in pulse thermography experiments not only for circular cracks as reported in [3] but also for flat bottom holes (in this case the slope of straight lines obviously do not coincide with the one indicated by eq.(2)).

In spite of this it remains to explain what contributions have been neglected in theoretical $2 \mathrm{D}$ models so that a mismatching between the theoretical and experimental FWHM is obtained.

\section{2. $2 \mathrm{D}$ model analysis}

As discussed in detail in [5], both models make the approximation that multiple reverberations occur as plane wave reflections between the sample surface and the defect, while this is strictly true only for the first reflection. In order to eliminate this simplification a suitable diaphragm on the defect plane was introduced so that a new integral equation has been obtained (see eq. (5) [5]). Using this modified model, FWHM as a function of time was studied and the results reported in Fig. 1. It can be noted that also in this case the curve fitting of the computed data is very similar to the previous ones. Moreover, the comparison between the trends of FWHM as a function of time for models with either single or multiple reverberations give evidence of the weight of multiple reverberations in correctly sizing the defect.

\subsection{Heat losses}

In all the considered models the heat losses have been neglected. In order to study convective losses contributions to the time dependence of FWHM, TERMO.HEAT ${ }^{\text {TM }}$ (a finite differences program) was used for simulating the trend of FWHM considering these losses. The results, shown in Fig. 1, also cannot explain the experimentally obtained trend of FWHM. It is interesting to observe that these results agree with the ones presented in literature [8]. A general comment on the curves shown in Fig. 1 is that the minimum value of FWHM for all these curves corresponds to the time of maximum contrast. Comparing the FWHM versus time curves corresponding to numerical simulation with and without convective losses it is possible to observe that they differ only by a negligible amount. This can be justified remembering that the maximum temperature difference between the sample surface over the defect and over a sound region occurs a few instants after the heat pulse while convective losses usually need longer times to reach their maximum effect. Furthermore, the aforementioned temperature difference typically has a maximum value of few. degrees $\left(<3^{\circ} \mathrm{C}\right)$; this means that the difference of convective heat losses between sound region and defect is really negligible.

In order to study experimentally convective loss effects, pulse thermography measurements have been carried out subjecting the surface to a forced convection produced by a fan (without air heating) placed near to the sample surface. A comparison between the time dependence of FWHM in this experimental condition and in the previous one is reported in Fig. 2. Obviously, the slopes of the two curves do not differ significantly (if the standard deviation of both slopes is taken into account). However, it seems that forced convection increases the slope of the curve, hence, also in this case, data can be fitted with a straight line.

\subsection{Noise and temperature resolution of the thermographic camera}

Theoretical models indicate that for times longer than the maximum contrast the FWHM should assume values higher than the true defect size. Another possible cause of the mismatch between theory and experiment could be related to the temperature resolution of the IR camera. In order to estimate the possible effect of the limited temperature resolution and other noise sources, a Gaussian noise was added to the theoretical profiles of the 
thermal contrast A simulation of theoretical contrast profiles showed that this effect is also negligible (see Fig. 1).

\subsection{Extension of the sizing procedure to irregular shaped defects}

This method is based on the following consideration: in an infrared image of a sample surface (with uniform emissivity) at a uniform temperature $T_{0}$, the pixel distribution as a function of the temperature has a Gaussian shape centred in $T_{0}$. [9] After the heat pulse (in a pulse thermography test) at each instant we suppose that the previous statement is true if there are no defects. On the contrary, the presence of a sub-surface defect within the sample modifies this Gaussian distribution because defect usually appears as a hot spot. For each IR image, the measurement of the number of pixel not belonging to the non distorted Gaussian distribution (which refers to the pixel distribution of a sound area) allows the estimation of a succession of apparent defect areas. Then in order to evaluate the true area, these apparent areas can be fitted by a straight line similarly to the case of circular shaped defects. In fact, if for circular defects FWHM exhibits a linear dependence on the square root of time, the same should relate to a whole defect area. This assumption is extended also to irregularly-shaped defects. This sizing procedure was applied to a flat bottom hole (10 $\mathrm{mm}$ diameter at $0.5 \mathrm{~mm}$ depth) and to nearly square $(10 \times 10 \mathrm{~mm})$, rectangular $(5 \times 6 \mathrm{~mm})$ and circular $(10 \mathrm{~mm}$ dia.) artificial disbonding defects in samples coated with metallic $(150 \mu \mathrm{m}$ NiCrCrAlY coating deposited by vacuum plasma spray) and ceramic (250 $\mu \mathrm{m}$ $\mathrm{ZrO}_{2}+8 \mathrm{wt} \% \mathrm{Y}_{2} \mathrm{O}_{3}$ deposited by air plasma spray) materials respectively. The experimental results (see Fig.3), confirm that this procedure allows estimating the area of a non-regular shaped subsurface defect. However, further experimental activities on other samples containing more irregular defects should be carried out.

\section{Conclusions}

The mismatch between theoretical and experimental FWHM trends as a function of time has been studied and a possible explanation has been found. Moreover the new sizing procedure proposed from the first experimental results seems to be well suited also for non circular defects.

\section{Acknowledgments}

The authors want to thank Dr. F. Lakestani of Institute for Advanced Material -Joint Research Centre (Ispra -Italy) for his fruitful suggestions and $E$. Colombo and V. Russo of Politecnico di Milano - Italy for their contribution at the beginning of this work.

\section{REFERENCES}

[1] VAVILOV (V.) - TOMOS: Thermal Characterization of Material Defects Using Transient Infrared Thermography. - Innovation Inc. 1996.

[2] MALDAGUE (X.) - Nondestructive evaluation of materials by infrared thermography. Springer Verlag, London 1993.

[3] ALMOND (D. P.) and LAU (S. K.) - Defect sizing by transient thermography. I: an analytical treatment. - J. Phys. D: Appl. Phys. 27, 1994 p.1063-1069.

[4] WETSEL (G. C.) and MCDONALD (F. A.) - Resolution and definition in photothermal imaging. - J. Appl. Phys. 56, 1984 p.3081-3085.

[5] CERNUSCHI (F.) LUDWIG (N.) and TERUZZI (P.) - A critical analysis and possible modifications of two analytical models for defect sizing using Video Pulse Thermography. - Presented at QIRT'98, Eurotherm Seminar n60 1998. 
[6] CROWTHER (D. J.), FAVRO (L. D.), KUO (P. K.) and THOMAS (R. L.) - Inverse scattering algorithm applied to infrared thermal wave images. - J. Appl. Phys. 74, (9), 1993 p. 5828-5834.

[7] LAU (S. K.), ALMOND (D. P.) and MILNE (J. M.) - A quantitative analysis of pulsed video thermography. - NDT\&E International, Vol.24 n 41991 p. 195-202.

[8] SAINTEY (M. B.) and ALMOND (D. P.) - Defect sizing by transient thermography III: a numerical treatment. - J. Phys. D: Appl. Phys., 281995 p. 2539-2546.

[9] CERNUSCHI (F.), COLOMBO (E.), RUSSO (V.), SALERNO (A.), GHIA (S.) and MARCHESI (R.) - Ceramic coating non destructive quality control by using video pulse thermography - Tranchina (B. S.) and. Bellosi (A.) eds. 4th EURO-CERAMICS - Coatings and Joinings Vol. 9, 1995, p. 419-428.

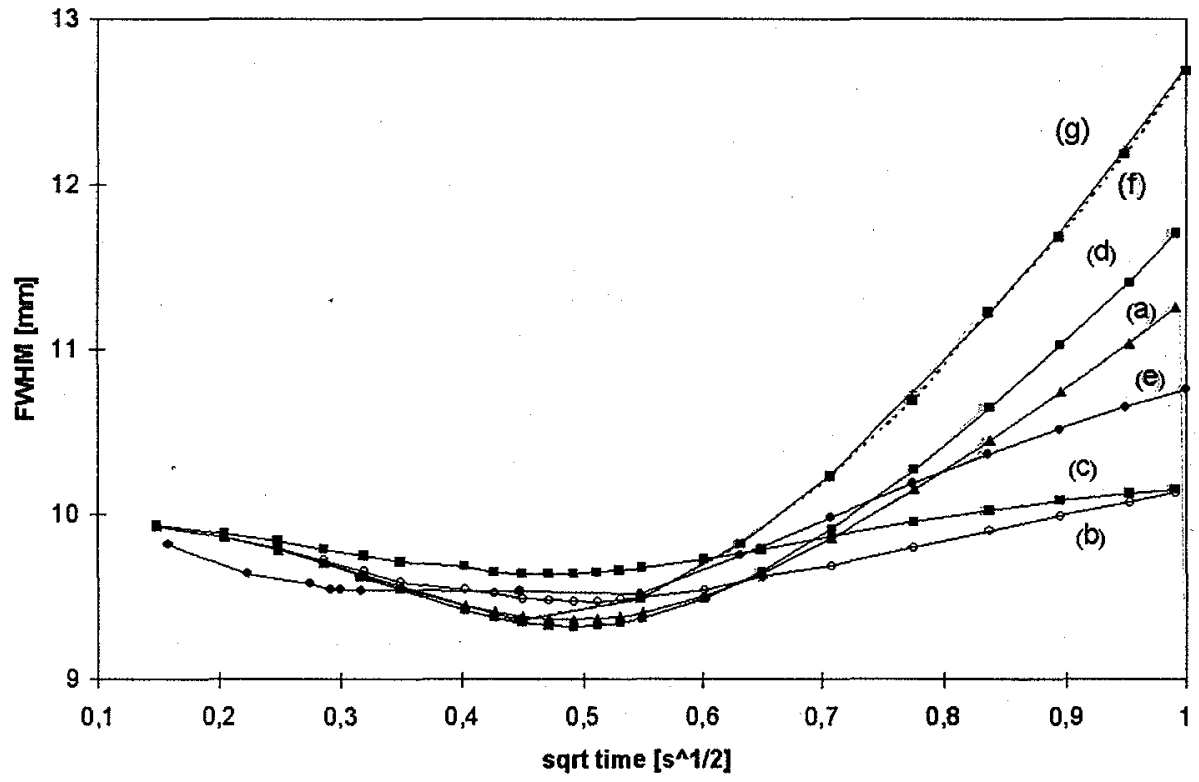

Fig. 1. FWHM of thermal contrast $C(x, t)$ versus square root of time. Curves were evaluated for mild steel $\left(\alpha=0.14 \cdot 10^{-4} \mathrm{~m}^{2} / \mathrm{s}\right.$ and $\left.\varepsilon=17100 \mathrm{~J} / \mathrm{m}^{2} \mathrm{~s}^{1 / 2} \mathrm{~K}\right)$ sample with a flat bottom hole (depth $L=1 \mathrm{~mm}$, radius $a=5 \mathrm{~mm}$ ) and for a heating energy density $I_{0}=10000 \mathrm{~J} / \mathrm{m}^{2}$. Curves refer to Almond's model for (a) one and (b) multiple reverberations, Crowther's model for (c) one and (d) multiple reverberations, (e) diaphragm model, (f) considering a Gaussian noise superimposed to the thermal contrast profile and TERMO.HEAT ${ }^{\mathrm{TM}}$ simulations $(\mathrm{g})$ without heat losses and $(\mathrm{h})$ with heat losses $\left(h=100 W / m^{2} K\right)$. 
http://dx.doi.org/10.21611/qirt.1998.003

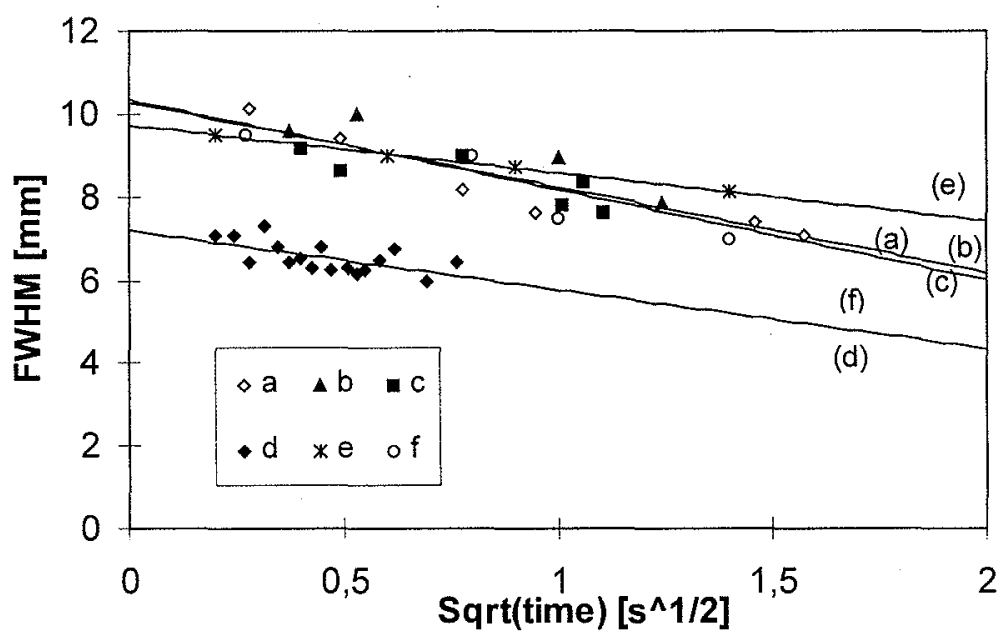

Fig. 2. FWHM of the thermal contrast $C(x, t)$ versus the square root of time. Curves refer to $10 \mathrm{~mm}$ diameter flat bottom holes at (a) $0.5 \mathrm{~mm}$ depth (b) $1 \mathrm{~mm}$ depth (c) $1 \mathrm{~mm}$ depth with forced convection (d) $7 \mathrm{~mm}$ diameter at $0.5 \mathrm{~mm}$ depth, $10 \mathrm{~mm}$ diameter (e) $1 \mathrm{~mm}$ depth and (f) $1 \mathrm{~mm}$ depth with the hole partially bunged. Curves (a)-(d) refer to mild ferritic steel samples while curves (e)-(f) refer to a nickel-based alloy sample.

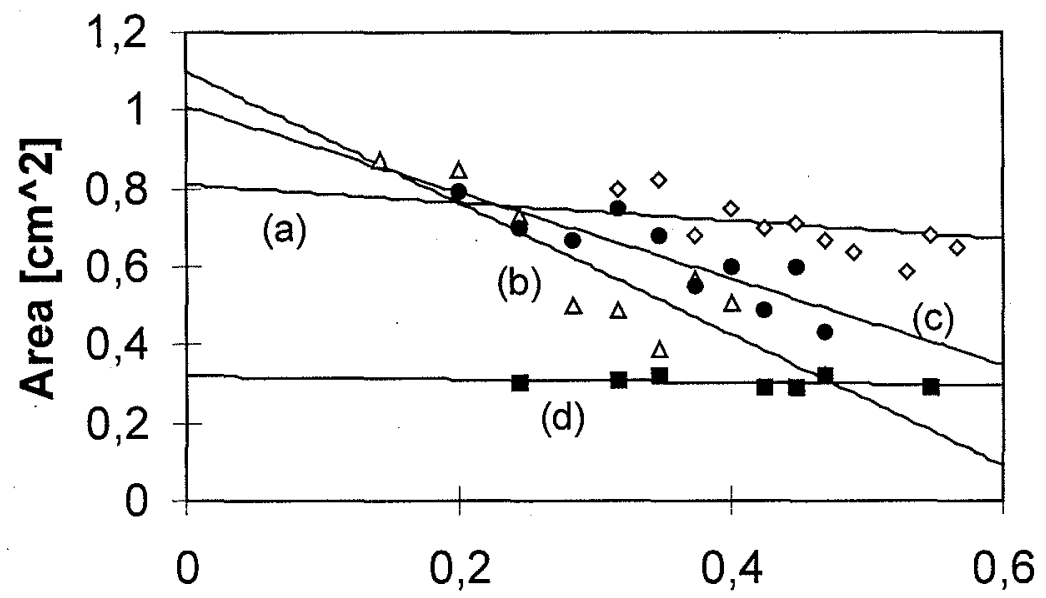

\section{Sqrt(time) [s^1/2]}

Fig. 3. Area versus square root of time for (a) $10 \mathrm{~mm}$ diameter flat bottom hole at $1 \mathrm{~mm}$ depth in a ferritic mild steel sample, square (b) and (c) circular disbonding defect in an AISI316 sample coated with a metallic coating and (d) a rectangular disbonding defect in an AISI316 sample coated with a ceramic coating. 